\title{
Development of head protector for judo practice considering neck extension suppression
}

\author{
Takeshi KAMITANI*,***, Natsuko ONIDANI*, Masaki OMIYA** and Atsuhiro KONOSU**** \\ *School of Integrated Design Engineering, Graduate School of Science and Technology, Keio University \\ 3-14-1 Hiyoshi, Kohoku-ku, Yokohama, Kanagawa, 223-8522, Japan \\ E-mail: takeshikami0806@yahoo.co.jp \\ **Department of Mechanical Engineering, Faculty of Science and Technology, Keio University \\ 3-14-1 Hiyoshi, Kohoku-ku, Yokohama, Kanagawa, 223-8522, Japan \\ *** Department of Orthopaedics, Japan Community Health Care Organization, Tokyo Shinjuku Medical Center \\ 5-1 Tsukudo-cho Shinjuku-ku, Tokyo, 162-8543, Japan \\ **** Japan Automobile Research Institute \\ 2530 Karima, Tsukuba, Ibaraki, 305-0822, Japan
}

Received: 18 May 2017; Revised: 19 October 2017; Accepted: 13 December 2017

\begin{abstract}
Severe head injuries - and even deaths - have been reported in the practice of judo, often resulting from inexperience in using the appropriate falling technique (ukemi). In this study, we developed a novel head protector that protects the head and suppresses the neck extension during impact with the mat. The head protector was fabricated for this purpose and consists of a head support structure and shock-absorbing materials. The effectiveness of the head protector was tested experimentally with an apparatus with head and neck components that were based on a Hybrid III dummy. Several configurations of the support structure characteristics and the shock-absorbing materials were evaluated. The proposed head protector was capable of reducing the head angular velocity variation range-which is an important evaluation index for the onset of acute subdural hematoma - by $29 \%$ using the devices alone, by $21 \%$ using shock-absorbing materials alone, and by $47 \%$ using a combination of both. These results show that the proposed structures are highly effective in suppressing extensions at the cervical-thoracic joint (C7-T1), whereas the shock-absorbing materials are highly effective in suppressing rotations at the atlanto-occipital joint (O-C1). It is concluded that wearing the proposed head protector - which combines both effects - can reduce the risk of severe head injuries, even those resulting from severe impacts such as those associated with the judo ukemi practice.
\end{abstract}

Keywords : Judo, Neck extension, Head protector, Acute subdural hematoma, Shock absorption

\section{Introduction}

Severe head injuries are often observed in various sports, such as American football (Cantu and Mueller, 2000, Boden et al., 2007), snowboarding (Prall et al., 1995; Fukuda et al., 2001), and baseball (Boden et al., 2004). In particular, severe head injuries in judo-leading to death or advanced sequelae-have been reported in Japan, where this sport originated (Nagahiro et al., 2011; Kamitani et al., 2013). The prevention of these injuries has been well recognized as a critical issue in recent years.

More than $90 \%$ of the severe head injuries are acute subdural hematomas (ASDH) with no apparent damage to the brain parenchyma; often, beginners develop symptoms following bruising of the occipital area (Nagahiro et al., 2011; Kamitani et al., 2013). In head impacts resulting from the judoka being thrown to the mat, a relative displacement between the brain and skull occurs because inertial forces lead to a lag in the rebound of the brain parenchyma compared to that of the skull. ASDHs likely occur as a result of this sudden rebound of the head, caused by ruptures in the bridging veins that cross the subdural space (Nagahiro et al., 2011). Fatalities have been reported in cases where the beginner judoka developed ASDH after experiencing occipital impacts in ukemi practice (Kamitani et al., 2013). One 
factor often cited for such injuries is the use of the unskilled falling technique (ukemi) (Nagahiro et al., 2011; Kamitani et al., 2013). In fact, neck extensions and large angular head rotation problems have been noted in experiments designed to analyze the body movements of beginners executing the $u$ kemi technique (Kamitani et al., 2014, Koshida et al., 2017).

Many types of head protectors or head gears designed to protect the head have been studied (Macnab et al., 2002; Brent et al., 2005; Hagel et al., 2005; Muller et al., 2008; Engsberg et al., 2008, Cusimano and Kwok, 2010, Ishikawa et al., 2013). However, these protectors mainly focus on the deceleration of the head during impact. Neck extension leads acceleration of the head and induces rotational motion of the head after falling backward on the mat. Therefore, it may be effective to suppress the extension of the neck to reduce the acceleration and rotational motion of the head, which has a chance to reduce the risk of ASDH occurrence significantly. Thus, the purpose of this study is to develop a judo head protector that protects the head and suppresses the neck extension, and to evaluate the effectiveness of thereof. In this present study, the angular velocity variation range which is the most effective injury index for ASDH was used in the evaluation. In addition, head angle, neck angle, maximum neck angular velocity, and head rebound angle were also used in the evaluation supplementary.

\section{Experimental methods}

A head protector was developed for beginners for use in their practice of the $u k e m i$ technique to prevent a head injury by considering the aforementioned unique characteristics of the ukemi movement in beginners. The protector consists of a neck-extension suppression device to constrain neck movement, and shock-absorbing materials to absorb head impacts (Fig. 1(a)-(c)). The key characteristic of the proposed head protector, specifically developed for judo practice, is that it not only protects the head, but also suppresses neck extensions.

The neck-extension suppression device was designed based on the 3-point support theory (Jozefiak-Wojtowicz, 2014); supports were placed at the occipital bone, sixth thoracic vertebra, and forehead. The material used in this device was thermoplastic resin with three different thicknesses: $3 \mathrm{~mm}(\mathrm{~A}), 6 \mathrm{~mm}(\mathrm{~B})$, and $9 \mathrm{~mm}(\mathrm{C})$. Three types of 10-mm thick shock-absorbing material were used: airpad (1), low resilience urethane (2), and medium-density polyurethane foam (3). The shock-absorbing material was placed on the occipital area, attached with velcro (see Fig. 1(c)), and was then fixed to the trunk using a band integrated with the neck extension suppression device (Fig. 1(a)(c)).

The head protector was evaluated using the experimental equipment shown in Fig.2. This experimental setup is based on the size of an average junior high school boy in first grade, and models the falling backward from Sonkyo (squatting posture), as shown in Fig.1. The weight of the experimental equipment is $44.5 \mathrm{~kg}$ and the height is $1030 \mathrm{~mm}$, with the center of gravity at a height of $462 \mathrm{~mm}$ from the center of rotation. The head and neck components were based on the Hybrid III dummy ( $50^{\text {th }}$ percentile adult male in the USA). The neck angle was set at $21.5^{\circ}$ forward inclination, which closely approximates the flexion position while performing ukemi. The trunk part comprises steel square pipes, and a pad of shock-absorbing material (5-mm thick gel sheet with a $200 \times 300 \mathrm{~mm}^{2}$ shock absorbing surface) is attached to it, so that the tatami mats are not damaged. The experiments are performed using a tatami mat (27TT-40004, Mizuno Corp.) for judo as prescribed by the International Judo Federation.

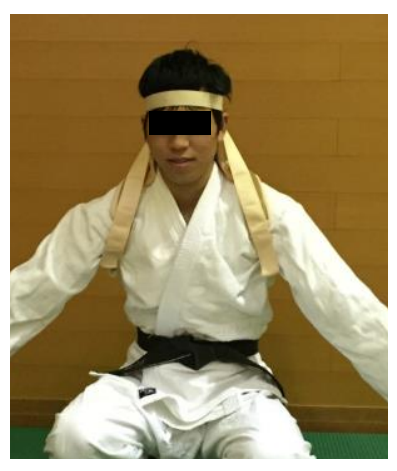

(a) Front view

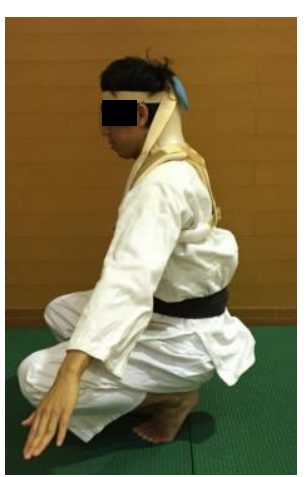

(b) Lateral view

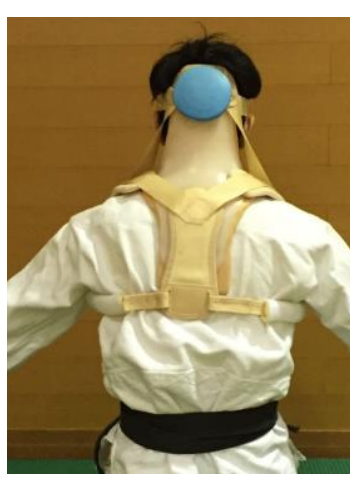

(c) Rear view

Fig. 1. Judo head protector comprising a central support structure (device) and shock-absorbing materials 


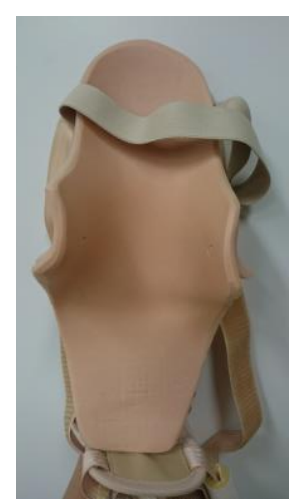

(a) Front view

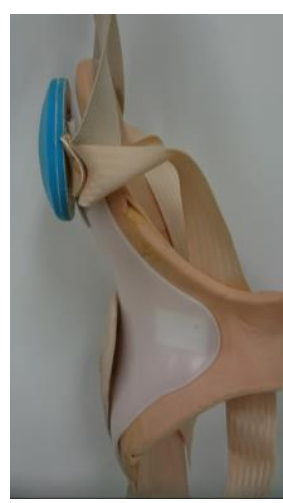

(b) Lateral view

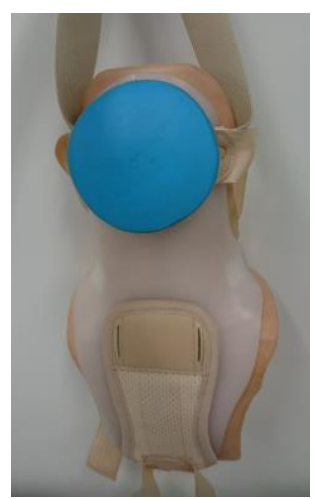

(c) Rear view

Fig. 2. Judo head protector

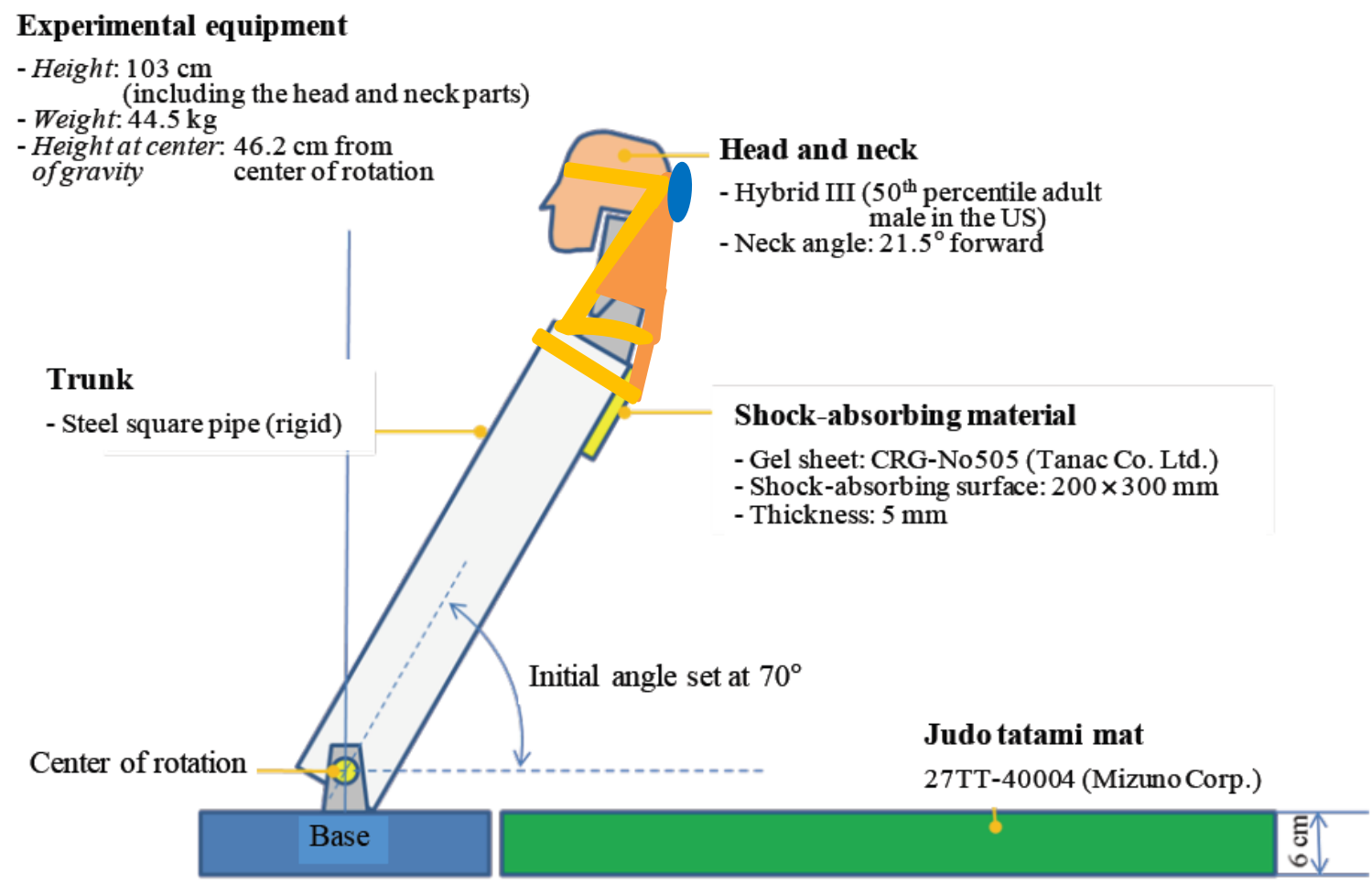

Fig. 3. Experimental setup and test conditions. A Hybrid III dummy was used for the head and neck components, and a steel square pipe was used for the trunk. The angle with respect to the horizontal from which the apparatus was dropped was set at $70^{\circ}$, to simulate a squatting posture. The speed upon impact was adjusted to approximately $2.19 \mathrm{~m} / \mathrm{s}$.

The velocity upon impact was set at $2.19 \mathrm{~m} / \mathrm{s}$, in accordance with previous ukemi impact experiments (Kamitani et al., 2014). Thus, the apparatus was set at $70^{\circ}$ with respect to the horizontal plane, and then dropped on the tatami mat to simulate falling backward. The experiments for the no-protector case were repeated two times to check the reproducibility of the experimental results.

Each experiment was performed with the three different devices and three shock-absorbing materials, independently or in combination; the baseline was the use of no head protector at all. The angular velocity variation 


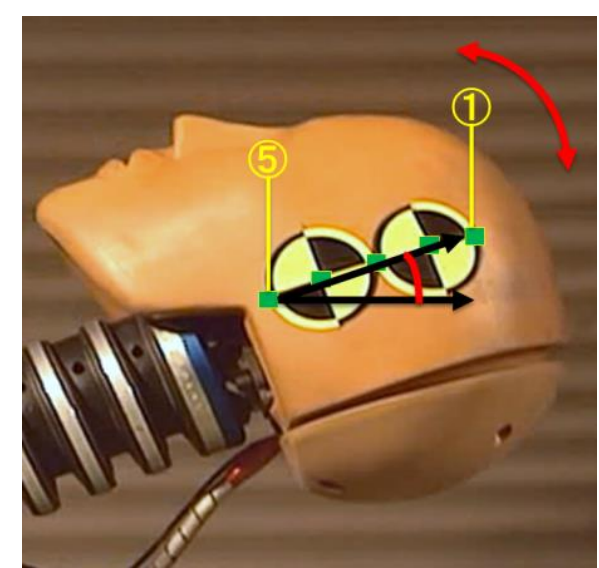

(a) The head angle was defined as the angle between the horizontal plane and vector $\boldsymbol{v}_{15}$ (connecting tracking points 1 and 5). Vector $\boldsymbol{v}_{15}$ is calculated from points $1-5$ using the least squares method.

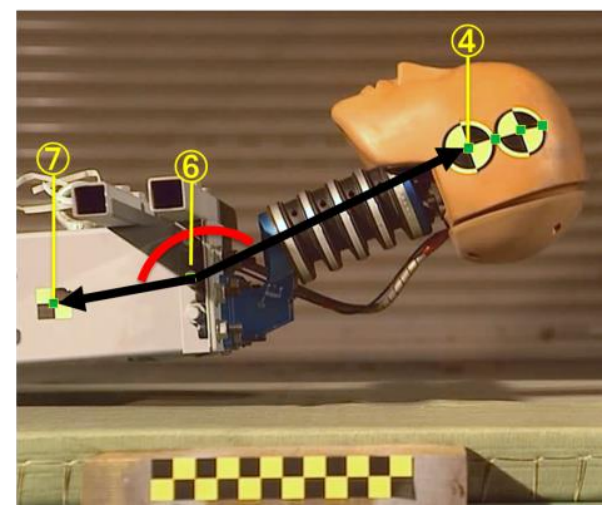

(b) The neck angle was defined as the angle between vector $\boldsymbol{v}_{46}$ (connecting tracking points 4 and 6) and vector $\boldsymbol{v}_{67}$ (connecting tracking points 6 and 7).

Fig. 4. Definition of the head and neck angles.

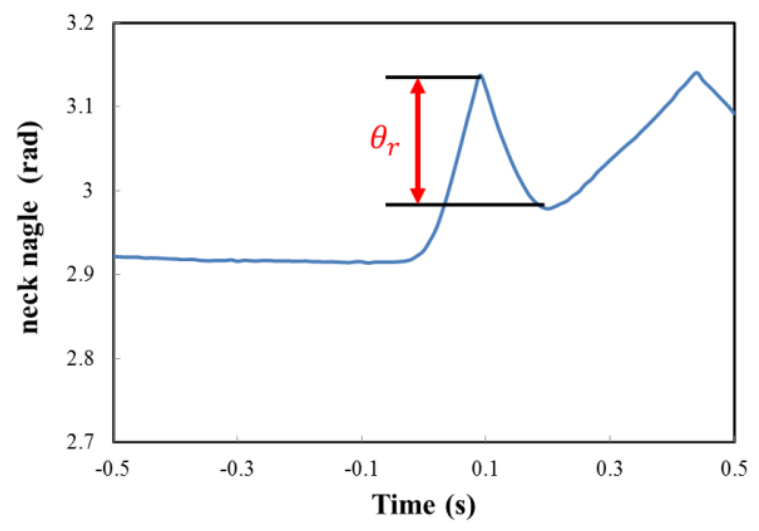

Fig. 5. Definition of the head rebound angle $\left(\theta_{\mathrm{r}}\right)$. This angle is defined as the difference between the maximum neck extension angle and the maximum neck flexion angle, and represents the rebound movement of the head.

range - which is an effective injury index for ASDH - was used in the evaluation of the head protector effectiveness (Aiba et al. 2012). The head angular velocity variation range was calculated as the difference between the maximum and minimum angular velocities measured using the angular velocity sensor (ARSPRO-18K; DTS Inc.) placed in the head of the Hybrid III dummy.

The head and neck angles were calculated by image analyses. The motion was captured using a high-speed camera (MEMRECAM GX-1; NAC Corp.; speed: 1000 fps, resolution: $1280 \times 1024)$. The captured images were analyzed 
Table 1 Normalized angular velocity variation range. In stand-alone tests, device $\mathrm{C}$ was the most effective. The combined use of device $\mathrm{C}$ and shock-absorbing materials resulted in a reduction of approximately half of the normalized value.

\begin{tabular}{|c|c|c|c|c|}
\hline $\begin{array}{c}\text { Shock-absorbing } \\
\text { material }\end{array}$ & None & $(1)$ & $(2)$ & $(3)$ \\
\hline None & 1.0 & 0.91 & 0.82 & 0.79 \\
\hline A & 0.82 & 0.77 & 0.66 & 0.59 \\
\hline B & 0.88 & 0.77 & 0.74 & 0.66 \\
\hline C & 0.71 & 0.66 & 0.63 & 0.52 \\
\hline
\end{tabular}

Table 2 Maximum neck angular velocity. In tests using only the device, the maximum neck angular velocity was reduced, especially with device $\mathrm{C}$. When devices and shock-absorbing materials were used together, the combination of device $\mathrm{C}$ and shock-absorbing material (3) resulted in the largest reduction.

\begin{tabular}{|l|c|l|l|l|}
\hline & $\begin{array}{c}\text { No } \\
\text { shock-absorbing } \\
\text { material }\end{array}$ & $(1)$ & $(2)$ & $(3)$ \\
\hline No device & 29.4 & 28.1 & 28.1 & 24.4 \\
\hline Device A & 22.7 & 25.2 & 21.6 & 20.0 \\
\hline Device B & 23.3 & 25.0 & 25.9 & 19.6 \\
\hline Device C & 20.5 & 21.2 & 21.1 & 18.6 \\
\hline
\end{tabular}

using the PV Studio 2D software package. In the analysis of the head and neck angles, the moment of trunk impact was defined as the origin of time $(0 \mathrm{~s})$.

Seven markers were attached to the dummy as shown in Fig.4, and points 1-7 (starting from the head side) were tracked in the image analyses. The head angle, which is the angle between the horizontal plane and vector $\boldsymbol{v}_{15}$ connecting tracking points 1 and 5, represents the rotation at the atlanto-occipital joint (O-C1) (Fig. 4(a)). The least squares method was used to estimate the angle between the horizontal plane and the line connecting tracking points $1-5$ (Fig. 4(a)). The neck angle was defined as the angle between vector $\boldsymbol{v}_{46}$ (connecting tracking points 4 and 6) and vector $\boldsymbol{v}_{67}$ (connecting tracking points 6 and 7), and it represents the cervical-thoracic joint (C7-T1) extension (Fig. 4(b)). In addition, the head rebound angle was measured to assess the head resilience behavior. This angle is defined as the difference between the maximum neck extension angle and the maximum neck flexion angle (see Fig. 5).

In the experiments conducted without the head protector, the observed variation range of the measured angular velocity was approximately $80 \mathrm{rad} / \mathrm{s}$. This is approximately twice the standard impact threshold for subdural hematoma onset (41 rad/s)(Löwenhielm, 1974, Nakamachi et al., 2003, Depreitere, 2005). Considering the actual head impacts experienced by beginners practicing ukemi, the experimental conditions were severe; this is intentional. By choosing such severe impact conditions as a baseline for development of the head protector, the developed product can be safely used as an effective protection against high-risk high-consequence injuries.

\section{Experimental Results}

\subsection{Angular velocity variation range}

The angular velocity variation range is an effective injury index for ASDH and is used in the evaluation of the head protector effectiveness. The experimental results obtained for the various cases are shown in Fig. 6: no protection, stand-alone use of devices $\mathrm{A} / \mathrm{B} / \mathrm{C}$, stand-alone use of shock-absorbing materials (1)-(3), and the combined use of devices $\mathrm{A} / \mathrm{B} / \mathrm{C}$ and shock-absorbing materials (1)-(3). The angular velocity variation range values shown in Table 1 were normalized by the average value obtained in the no-protection case $(77.9 \mathrm{rad} / \mathrm{s})$.

From Table 1 , the tests using only protective devices resulted in 0.82 for device A, 0.88 for device $\mathrm{B}$, and 0.71 for device $\mathrm{C}$, demonstrating that device $\mathrm{C}$ was the most effective, with a reduction of $29 \%$ in the angular velocity variation range. The tests using only shock-absorbing materials resulted in values of 0.91 for material (1), 0.82 for material (2), and 0.79 for material (3), showing reductions of $19 \%$ and $21 \%$ for materials (2) and (3), respectively. In the tests where both a device and a shock-absorbing material were used, the combination of device $\mathrm{C}$ with material (3) showed the highest reduction in angular velocity range, with a normalized value of 0.52 (a $48 \%$ reduction). 


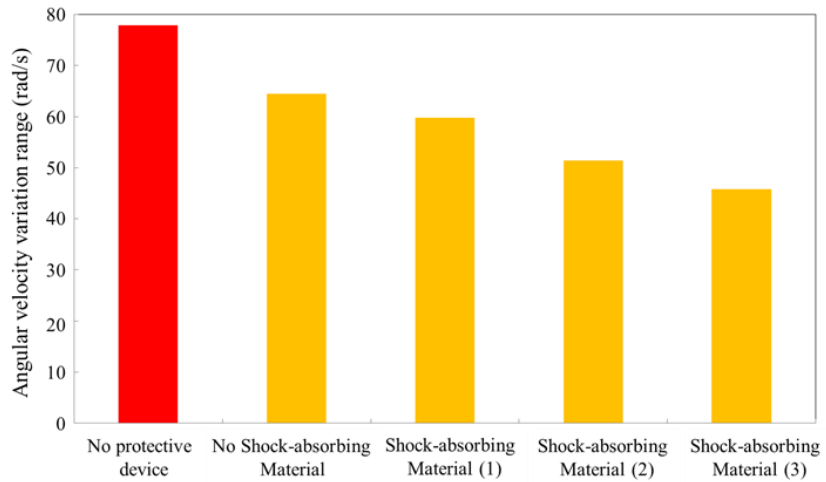

(a) Device A

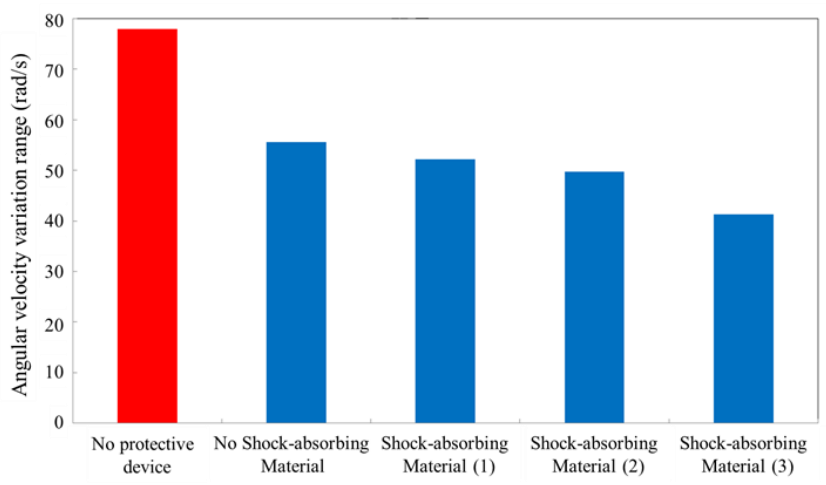

(c) Device C

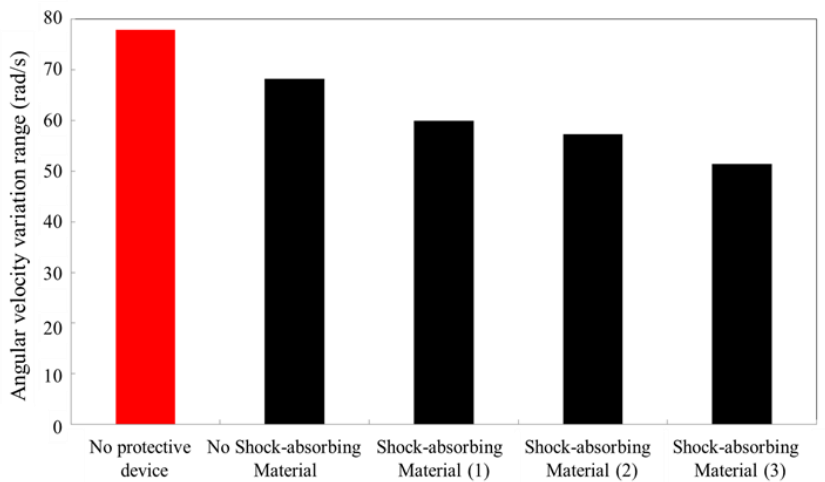

(b) Device B

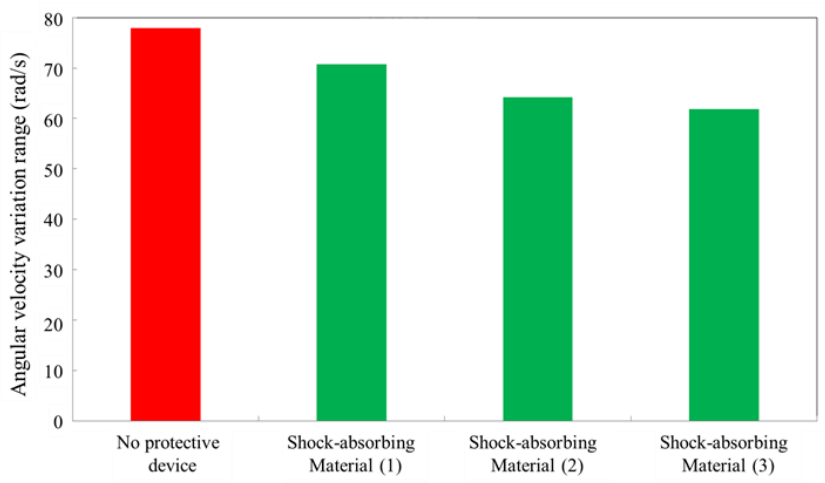

(d) Shock-absorbing materials only

Fig. 6. Angular velocity variation range. In the stand-alone tests, device $\mathrm{C}$ was found to be the most effective. When both devices and shock-absorbing materials were used together, the combination of device $\mathrm{C}$ with material (3) was found to be the most effective one.

\subsection{Head angle}

The head angle represents the rotation of atlanto-occipital joint $(\mathrm{O}-\mathrm{C} 1)$ and is related to the rotation of the head. The experimental results obtained for head angle are shown in Fig. 7(a)-(e). The results obtained in the three trials without protection are shown in Fig. 7(a). An occipital rebound occurred after head impact, which in turn occurred $0.1 \mathrm{~s}$ after the trunk hit the mat. The maximum head angle was $-0.278 \mathrm{rad}$, in the instant when the head hit the mat. It is confirmed that the experimental results are reproducible. The results obtained using the devices (both stand-alone and in combination with the shock-absorbing materials) are shown in Fig. 7(b)-(e). Figure 7(b) shows the results for device A, alone and in combination with materials (1)-(3); Figure 7(c) shows the corresponding results for device B, and Fig. 7(d) for device C. Finally, Fig. 7(e) shows the results obtained when only shock-absorbing materials were used.

In the case of the stand-alone use of device $\mathrm{C}$, the obtained maximum head angle was $-0.253 \mathrm{rad}$ (Fig. 7(d)), showing no significant difference to the average value of -0.260 rad obtained in the tests where no protection was used (Fig. 7(a)). In the tests with only shock-absorbing materials, material (2) resulted in a suppressed peak value of -0.134 $\mathrm{rad}$ (see Fig. 7(e)); with material (3), the resulting peak value was suppressed to $-0.223 \mathrm{rad}$. Furthermore, with material (3), the peak of the head angle waveform was effectively delayed, appearing only $0.32 \mathrm{~s}$ after the impact (see Fig. 7(e)), instead of the $0.11 \mathrm{~s}$ observed in the no-protection case. In the tests performed with a combination of devices and shock-absorbing materials, the combination of devices B and C with material (2) resulted in values of -0.146 and $-0.147 \mathrm{rad}$, respectively; thus exhibiting a reduction in the peak values (Fig. 7(c) and (d)).

\subsection{Neck angle}

The neck angle is related to the extension of cervical-thoracic joint (C7-T1). Figure 8(a)-(e) shows the results of the neck angle analysis. The results are slightly scattered owing to the tracking marker position picked by image analyses. Figure 8(a) shows the results obtained in the three trials without protection, where a maximum angle of 3.13 $\mathrm{rad}$ is reached approximately $0.1 \mathrm{~s}$ after head impact. Following this, as the head rebounded, the neck bent, thereby reducing the neck angle. However, at approximately $0.2 \mathrm{~s}$, a further neck extension occurred, resulting in a maximum 


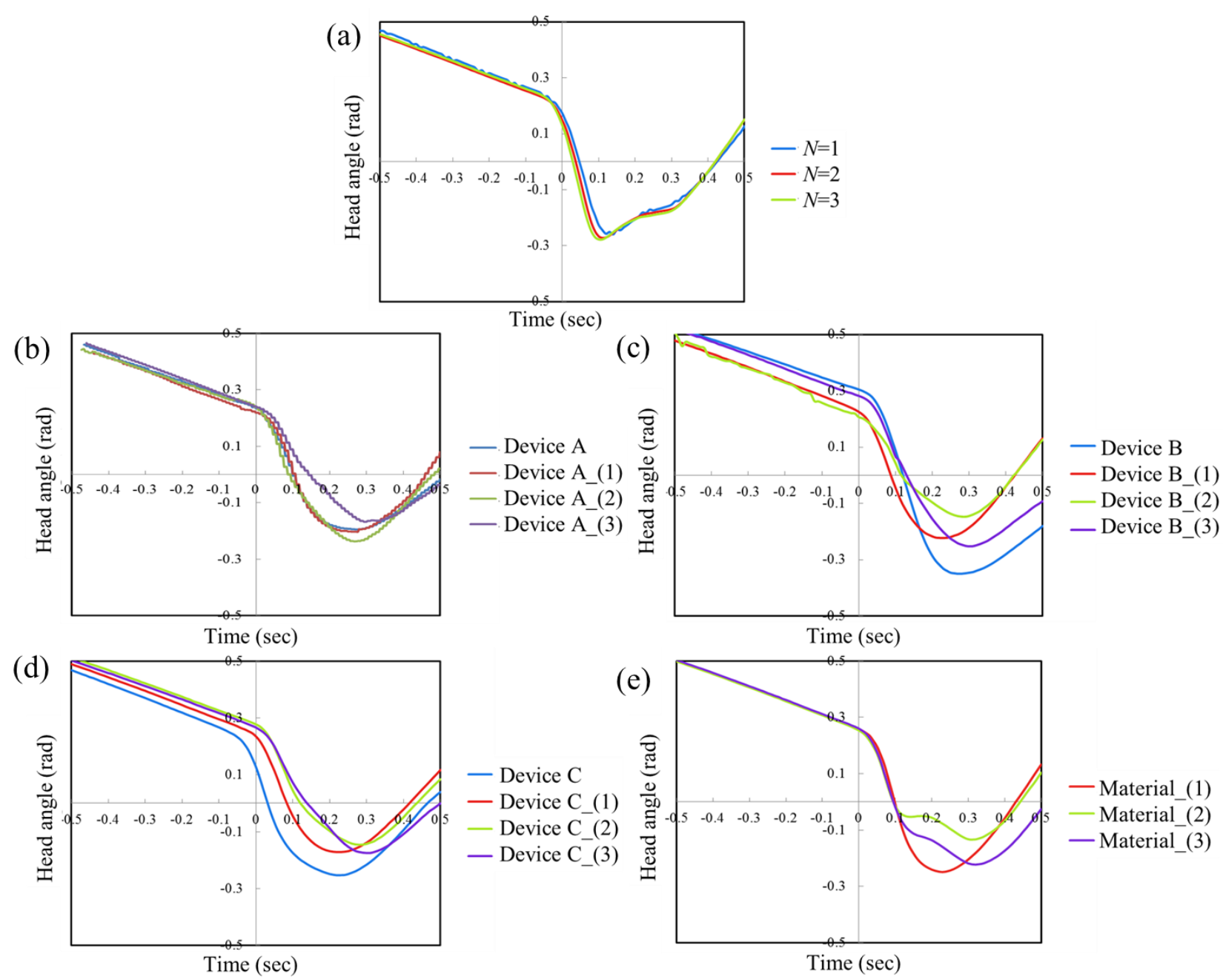

Fig. 7. Head angle; (a) no protective device, (b) Device A, (c) Device B, (d) Device C, (e) Shock-absorbing material only. Peak value suppression was observed in stand-alone use of shock-absorbing material (2). When the devices and shock-absorbing materials were used together, the combination of devices B and C with material (2) resulted in reducing the peak value.

angle of $3.13 \mathrm{rad}$ at approximately $0.3 \mathrm{~s}$. Figure $8(\mathrm{~b})-(\mathrm{e})$ was obtained under the same conditions as Fig. 7(b)-(e). In all cases (stand-alone devices, stand-alone shock-absorbing materials, and combinations thereof), neck extensions were observed up to a peak angle of $3.13 \mathrm{rad}$.

\subsection{Maximum neck angular velocity}

The neck angular velocities were calculated from the neck angles, and the results obtained for maximum neck angular velocity are shown in Table 2 . The neck angular velocity obtained using only device $\mathrm{C}$ showed a reduction to $20.5 \mathrm{rad} / \mathrm{s}$ (in contrast to $29.4 \mathrm{rad} / \mathrm{s}$ in the no-protection case). In the tests using only shock-absorbing materials, the values are $28.1 \mathrm{rad} / \mathrm{s}$ for material (1), $28.1 \mathrm{rad} / \mathrm{s}$ for material (2), and $24.4 \mathrm{rad} / \mathrm{s}$ for material (3). When device $\mathrm{C}$ and material (3) were combined, the angular velocity was further reduced to $18.6 \mathrm{rad} / \mathrm{s}$.

\subsection{Head rebound angle}

The head rebound angle is related to the motion of the skull, and the results obtained are shown in Figure 9. Without protection, the average value was $0.1 \mathrm{rad}$. In the tests using only protective devices, the obtained values were $0.147 \mathrm{rad}$ for device $\mathrm{A}, 0.103 \mathrm{rad}$ for device $\mathrm{B}$, and $4.50 \times 10^{-2} \mathrm{rad}$ for device $\mathrm{C}$; the lowest rebound was therefore observed with device $\mathrm{C}$. The tests with only shock-absorbing materials resulted in values of $6.44 \times 10^{-2} \mathrm{rad}$ for material (1), $6.66 \times 10^{-2} \mathrm{rad}$ for material (2), and $9.22 \times 10^{-2} \mathrm{rad}$ for material (3). When combinations of devices and shock-absorbing materials were used, significantly lower values were observed for device $\mathrm{C}$; in particular, for the combination of device $\mathrm{C}$ and material (3), which resulted in an extremely low observed value of $3.37 \times 10^{-3} \mathrm{rad}$. 

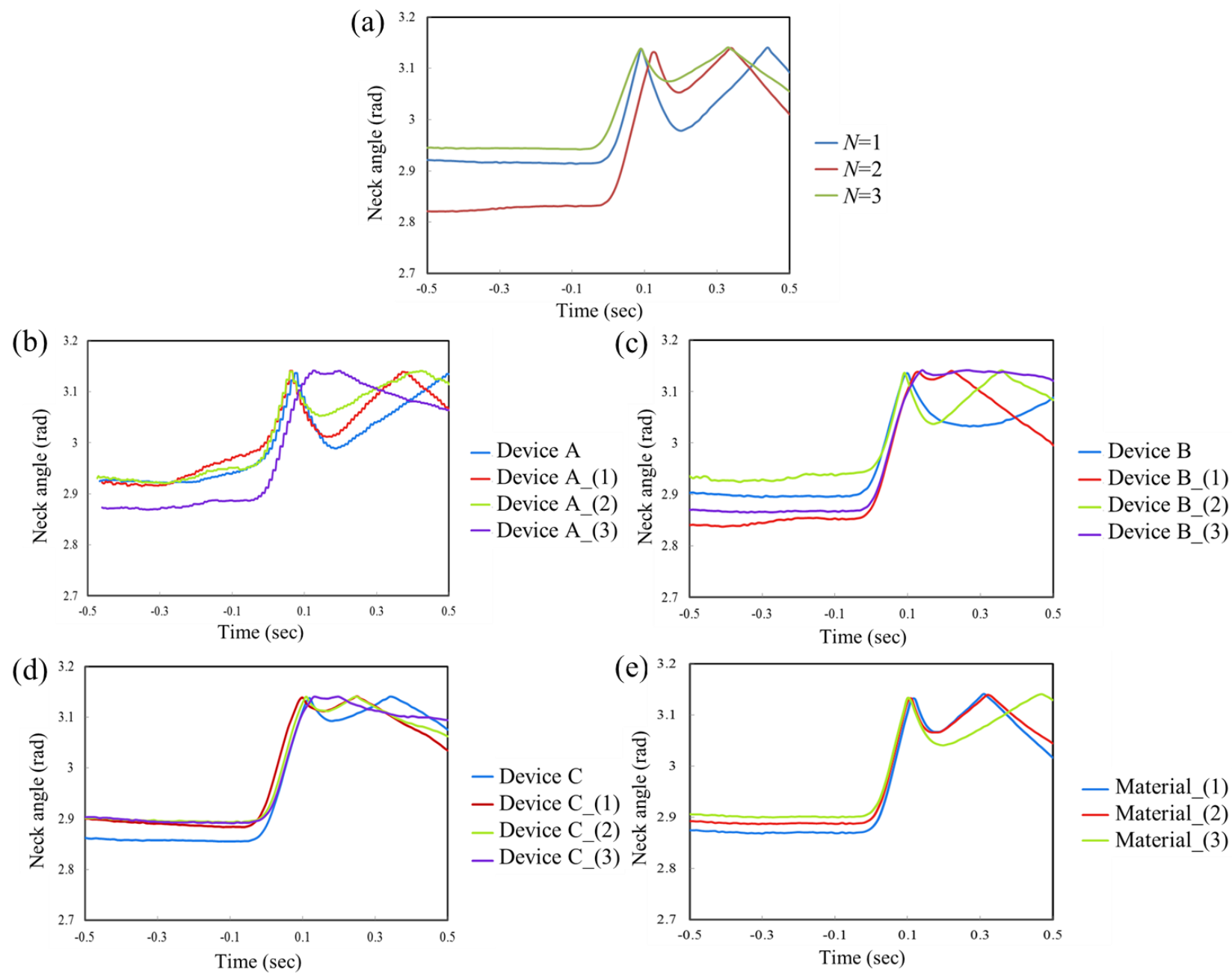

- Material_(1)
— Material_(2)
— Material_(3)

Fig. 8. Neck angle; (a) no protective device, (b) Device A, (c) Device B, (d) Device C, (e) Shock-absorbing material only. In all cases (stand-alone devices, stand-alone shock-absorbing materials, and combinations thereof), extensions up to a peak angle of $3.13 \mathrm{rad}$ were observed.

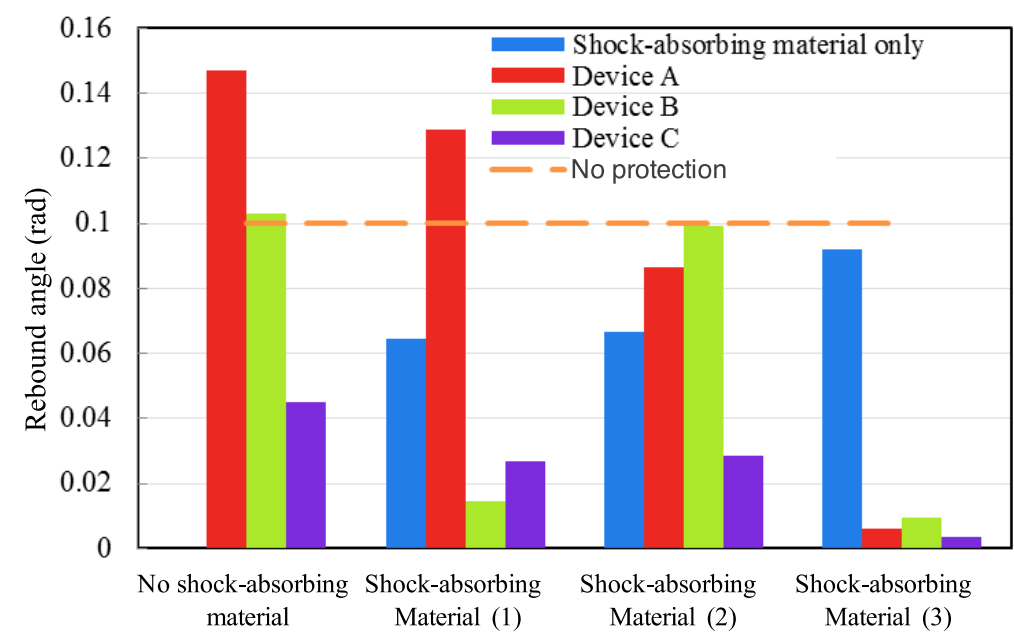

Fig. 9. Head rebound angle $\left(\theta_{\mathrm{r}}\right)$. Although the use of shock-absorbing materials alone was not found to be effective, the use of these materials combined with device $\mathrm{C}$ resulted in significantly lower values of head rebound angle. 


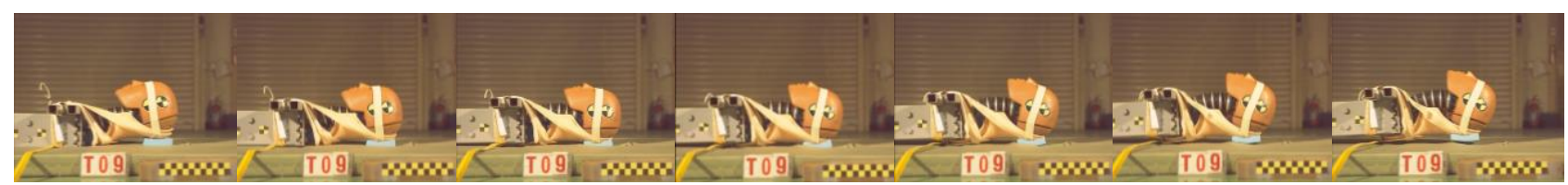

Fig. 10. the collision image in chronological order.

Figure 10 shows the collision image in chronological order.

\section{Discussion}

Several protective devices have been developed to prevent head injuries in sports medicine engineering studies (Macnab et al., 2002; Brent et al., 2005, Hagel et al., 2005), and their effectiveness has been analyzed frequently (Muller et al., 2008; Engsberg et al., 2008, Cusimano and Kwok, 2010). However, these devices protect only the head; their effectiveness in suppressing angular acceleration has not been clarified. Moreover, neck movement—which is one of the factors of soft impact causing ASDH-has not been studied.

The effectiveness of the proposed devices is discussed first. Without shock-absorbing materials, the use of device $\mathrm{C}$ resulted in a $29 \%$ reduction in the angular velocity variation range; device A (the thinnest of the three) reduced the angular velocity range by $18 \%$. In fact, the degree of neck extension suppression increased with increasing device thickness (see Fig. 6(a)-(c) and Table 1). However, when using only device C, the maximum head angle was -0.253 $\mathrm{rad}$ (see Fig. 7(d)), which is not significantly different from the average value of $-0.260 \mathrm{rad}$ observed in the no-protection case. In contrast, the maximum neck angular velocity was reduced markedly to approximately $20.5 \mathrm{rad} / \mathrm{s}$ when using only device $\mathrm{C}$, in comparison with the value of $29.4 \mathrm{rad} / \mathrm{s}$ for the no-protection case (see Table 2). These results suggest that this device is not highly effective in suppressing the rotation at atlanto-occipital joint $(\mathrm{O}-\mathrm{C} 1)$, but is highly effective in suppressing the extension at cervical-thoracic joint (C7-T1).

Suppression of the rotation at $\mathrm{O}-\mathrm{C} 1$ and the extension at C7-T1 are both necessary to increase the effectiveness of the neck extension suppression. The devices in this study have been developed based on medical cervical collars (Józefiak-Wójtowicz et al., 2014). The results of the present study suggest that it is preferable to extend the restraint even closer to $\mathrm{O}-\mathrm{C} 1$, to effectively suppress the rotation at that point. In addition, it is suggested that increasing the thickness of the thermoplastic resin used in the device may further suppress the extension at C7-T1. However, the thickness of device $\mathrm{C}$ is already $9 \mathrm{~mm}$, and it may be impractical to increase it further because this will likely make the device difficult to wear. Thus, light and stiff materials, such as fiber-reinforced composites (FRP), are expected to be candidates for device material.

The effectiveness of the shock-absorbing materials is discussed next. When using only shock-absorbing materials, the reductions in the angular velocity variation range were $9 \%$ with material (1), $18 \%$ with material (2), and $21 \%$ with material (3). The maximum neck angular velocities were $28.1 \mathrm{rad} / \mathrm{s}$ with (1), $28.1 \mathrm{rad} / \mathrm{s}$ with (2), and $24.4 \mathrm{rad} / \mathrm{s}$ with (3); these values are not significantly different from the observed value of $29.4 \mathrm{rad} / \mathrm{s}$ without any protection (see Table 1), suggesting that the shock-absorbing materials alone were not effective in suppressing neck angular velocity. However, using material (2) did suppress the peak value of the head angle to $-0.134 \mathrm{rad}$ compared to $-0.259 \mathrm{rad}$ for the no-protection cases (see Fig. 7(a) and (e)). Material (3) was not as effective in this regard, with a peak value of -0.223 $\mathrm{rad}$; however, it was effective in delaying the peak. These results suggest that the shock-absorbing materials are likely not highly effective in suppressing the extension at C7-T1, but are highly effective in suppressing the rotation at O-C1. Shock-absorbing materials (2) and (3) were more effective, which is probably because of their low coefficients of restitution and the shape retention property of the material (3).

Finally, the results of the tests where both a device and shock-absorbing material were used are now discussed. The combination of device $\mathrm{C}$ and shock-absorbing material (3) was highly effective, as it resulted in a $48 \%$ reduction in the angular velocity variation range. The head angle and maximum neck angular velocity were both suppressed. This combination also resulted in a significant reduction in the head rebound angle, showing a trend similar to that of the angular velocity variation range.

In summary, it can be said that the devices were highly effective in suppressing the extension at C7-T1, whereas the shock-absorbing materials were highly effective in suppressing the rotation at $\mathrm{O}-\mathrm{C} 1$. The results of the tests with 
combinations of both suggest that large reductions in the head angular velocity variation range and in the head rebound angle can likely be achieved using the appropriate combination. This study confirms that wearing the proposed head protector can reduce the angular velocity variation range by approximately a factor of two, even in severe impacts such as those found in the judo ukemi practice. Therefore, we believe that wearing this protector will enable the safe practice of $u$ kemi by judo beginners with weak neck muscles.

One concern in this study is that errors in the experimental results may have occurred because of gaps between the head/neck and the device. Further studies are required to design the shape of the device fitting the body shape. Moreover, the scatter observed in the head rebound angle measurements may likely have resulted from the viscous effects of the shock-absorbing materials. In this regard, a dynamical model is being studied.

It cannot be ignored that the use of a head and neck protector, such as the one proposed here, may likely increase the load on adjacent joints. Consequently, while such protectors may likely prevent the occurrence of ASDH, there is the possibility that new injuries are induced, such as cervical sprains. These aspects need to be considered in any future implementation of head and neck protective devices.

Although the protector developed is this study specifically addresses the practice of back ukemi, the authors hope to implement more diversified protective devices in the future by improving the devices and the shape and properties of the shock-absorbing materials, so that they can also be used in standing technique practices.

\section{Conclusions}

1) The proposed head protector with a mechanism to suppress neck extension was capable of reducing the head angular velocity variation range - which is an important evaluation index for the onset of ASDH-by $29 \%$ using the devices alone, by $21 \%$ using shock-absorbing materials alone, and by $47 \%$ using a combination of both. These results indicate that a combination of device and shock-absorbing material is a more effective choice.

2) The obtained results further suggest that the effectiveness of the head protector proposed in this study derives from the head rebound suppression obtained through the reduction in head angle and maximum neck angular velocity.

3) The proposed devices were highly effective in suppressing extensions at the cervical-thoracic joint (C7-T1), whereas the shock-absorbing materials were highly effective in suppressing rotations at the atlanto-occipital joint (O-C1). A combination of these effects results in increased effectiveness.

\section{Acknowledgements}

The authors are grateful for the support of Kenji Anata of JARI, Daiki Katori, Chikako Katori, and Suda Hidehiro of Katorigishi Inc., Taisuke Tomatsu (former chair, All Japan Judo Federation Medical Science Committee), and the students of Keio University.

\section{References}

Aiba, K., Omiya, M., Iteya, M., Kamitani, T. and Tomatsu, T., Comparison of Evaluation Indices for Acute Subdu-ral Hematoma Occurrence under Occipital Impact and Effectiveness of Head Protector in Judo, Journal of the Japan Society of Mechanical Engineers A, Vol.78, (2012), pp.1631-1641 (in Japanese), DOI:10.1299/kikaia.78.1631.

Boden, B. P., Tacchetti, R. and Mueller, F. O., Catastrophic injuries in high school and college baseball players, American Journal of Sports Medicine, Vol.32, No.5 (2004), pp.1189-1196, DOI:10.1177/0363546503262161.

Boden, B. P., Tacchetti, R. L., Cantu, R. C., Knowles, S. B. and Mueller, F. O., Catastrophic head injuries in high school and college football players, American Journal of Sports Medicine, Vol.35, No.7 (2007), pp.1075-1081, DOI: $10.1177 / 0363546507299239$.

Brent, H., Barry, P., Claude, G., Robert, P. and Yvonne, R., The effect of helmet use on injury severity and crash circumstances in skiers and snowboarders, Accident Analysis and Prevention, Vol. 37, No.1 (2005), pp.103-108, DOI:10.1016/j.aap.2004.04.003.

Cantu, R. C. and Mueller, F. O., Catastrophic football injuries: 1977-1998, Neurosurgery, Vol.47. No.3 (2000), pp.673-677.

Cusimano, M.D. and Kwok, J., The effectiveness of helmet wear in skiers and snowboarders: a systematic review, 
British Journal of Sports Medicine, Vol.44, No.11 (2010), pp.781-786, DOI:10.1136/bjsm.2009.070573.

Depreitere, B., Van Lierde, C., Verschueren, P., Delye, H., Berckmans, D., Verpoest, I., Vander Sloten, J., Van der Perre, G., Goffin, J.,Cerebral bridging vein rupture in humans, An experimental evaluation, IUTAM Proceedings on Impact Biomechanics: From Fundamental Insights to Applications, Michael D. Gilchrist (ed.),(2005),pp.305-312

Engsberg, J. R., Standeven, J. W., Shurtleff, T. L., Tricamo, J. M. and Landau, W. M., Spinal cord and brain injury protection: testing concept for a protective device, Spinal Cord, Vol.47, No.8 (2008), pp. 634-639, DOI:10.1038/sc.2009.1.

Fukuda, O., Takaba, M., Saito, T. and Endo, S., Head injuries in snowboarders compared with head injuries in skiers. a prospective analysis of 1076 patients from 1994 to 1999 in Niigata, Japan, American Journal of Sports Medicine, Vol.29, No.4 (2001), pp.437-440, DOI:10.1177/03635465010290040901.

Hagel, B., Pless, I. B., Goulet, C., Platt, R. and Robitaille, Y., The effect of helmet use on injury severity and crash circumstances in skiers and snowboarders, Accident Analysis and Prevention, Vol. 37, No.1 (2005), pp.103-108, DOI:10.1016/j.aap.2004.04.003.

Ishikawa, Y., Uchida, R. and Nagasaka, A., Reports for 2013 Mizuno Sports Promotion Foundation (2013), pp.1-12 (in Japanese).

Józefiak-Wójtowicz, A., Stolarczyk, A., Deszczyński, J. M., Materek, M., Pietras, M. and Bażant, K., Use of cervical collar after whiplash injuries. Polish orthophedics and traumatoly, Vol.79 (2014), pp.132-137.

Kamitani, T., Nimura, Y., Nagahiro, S., Miyazaki, S. and Tomatsu, T., Catastrophic head and neck injuries in judo players in Japan from 2003 to 2010, American Journal of Sports Medicine, Vol.41, No.8 (2013), pp.1915-1921, DOI: $10.1177 / 0363546513490662$

Kamitani, T., Mishima, S., Sonoda, M., Okada, S. and Omiya, M., Experimental investigation of motion analysis during back break falls in judo: a problematic point for beginners' ukemi, Japanese Journal of Clinical Sports Medicine, Vol.22 (2014), pp.325-330 (in Japanese).

Koshida, S., Ishii, T., Matsuda, T. and Hashimoto, T., Kinematics of judo breakfall for osoto-gari: considerations for head injury prevention, Journal of Sports Sciences, Vol.35, No.11 (2017), pp.1059-1065.

Löwenhielm P., Strain tolerance of the Vv. Cerebri sup. (Bridging veins) calculated from head-on collision tests with cadavers, Z. Rechtsmedizin, Vol.75, (1974), pp.131-144

Macnab, A. J., Smith, T., Gagnon, F. A. and Macnab, M., Effect of helmet wear on the incidence of head/face and cervical spine injuries in young skiers and snowboarders, Injury Prevention, Vol. 8, No. 4 (2002), pp.324-327.

Mueller, B. A., Cummings, P., Rivara, F. P., Brooks, M. A. and Terasaki, R. D., Injuries of the Head, Face, and Neck in Relation to Ski Helmet Use, Epidemiology, Vol.19. No.2 (2008), pp.270-276.

Nagahiro, S., Mizobuchi, Y., Hondo, H., Kasuya, H., Kamitani, T., Shinbara, Y., Nimura, Y. and Tomatsu, T., Severe head injuries during Judo practice, No Shinkei Geka. Neurological Surgery, Vol.39, No.12 (2011), pp.1139-1147 (in Japanese).

Nakamachi, E., Okuyama, T., Takegawa, Y., Omori, K., "Finite Element Analysis of acute subdural hematoma caused by rotational acceleration," Proceedings of $78^{\text {th }}$ JSME Kansai Branch conference, No. 034-1, (2003), pp.15-16 (in Japanese).

Prall, J. A., Winston, K. R. and Brennan, R., Severe snowboarding injuries, Injury, Vol.26, No.8 (1995), pp. 539-542. 